\title{
Disposable free three port laparoscopic appendectomy - low cost alternative for emergency
}

\begin{abstract}
Rationale: Video laparoscopic appendectomy does not have a single protocol on technical systematization, access routes, energy use, and staplers. The cost of disposable materials may prevent its widespread use. Alternatives to lower the cost can help disseminate laparoscopic access in appendectomy.

Objective: This study aimed to introduce a method in performing video laparoscopic appendectomy at low cost and aiming at a good aesthetic result through the location of its incisions and show its viability through its application in 1552 cases of video laparoscopic appendectomy performed between 2000 and 2019 with three portals, with extremely low cost in inputs used.

Methods: Three punctures were performed - one umbilical puncture to introduce the camera (5 or $10 \mathrm{~mm}$ in diameter), one $10 \mathrm{~mm}$ puncture in the right iliac fossa, and one 5 -mm puncture in the left iliac fossa. The last two punctures were performed medially to the epigastric vessels, which can be visualized with the aid of the laparoscopic camera externally, by transparency, or internally, under direct vision. The materials- trocars, grasping forceps, hooks, scissors, and needle holders, are of permanent use, without the need for any disposable material.
\end{abstract}

Results: A total of 1552 patients underwent operation between 2000 and 2019; 56.25\% were female, the mean age was 32.66years (9-93years), and the mean and median lengths of hospital stay were 1.74days (1-10days) and 1.2days, respectively.

Conclusion: The described technique uses three metal trocars and four permanent instruments, in addition to a single cotton thread. The use of extraction bags for operating parts, clips, handles, staplers or special energy, and bipolar or harmonic instruments was discontinued. Therefore, it is a low-cost laparoscopic procedure. Because it allows triangulation and instrumentation in the conventional way, it is a safe and reproducible surgery, which can be easily taught and widely used in hospitals that provide conventional laparoscopic equipment. Its application in 1552 patients in a 20 -year period has shown excellent results and low morbidity and may become routine with the preferred indication for video surgery in the treatment of acute appendicitis.

Keywords: appendectomy, laparoscopy, acute appendicitis
Volume II Issue 2 - 2020

\author{
Carlos Eduardo Domene, Paula Volpe, \\ Frederico Almeida Heitor, André Valente \\ Santana \\ Integrated Center for Advanced Medicine and Unified \\ Treatment Center for the Obese, Brazil
}

Correspondence: Carlos Eduardo Domene, A study conducted at the Integrated Center for Advanced Medicine and Unified Treatment Center for the Obese, São Paulo, Brazil, Email cedomene@terra.com.br

Received: March 08, 2020 | Published: April 28, 2020

\section{Introduction}

Appendicitis is a highly prevalent emergency disease with a lifetime incidence of about $8 \%$, and appendectomy is the most frequent surgery in emergency situations. ${ }^{1-4}$ McBurney open right lower quadrant incision was first described in 1894, and has been the most frequent access for open appendectomy since that time. Laparoscopic appendectomy, first performed back more than 30 years, has clear advantages over open approach: lower intra and postoperative complication rate, provides access to the whole abdominal cavity in order to inspect and aspire liquids, less postoperative pain and faster recovery. Despite these evidences, appendectomy is still performed with laparotomy in at least two-thirds of cases. ${ }^{5}$

The most common current laparoscopic techniques are as follows: three-port laparoscopic appendectomy, transumbilical laparoscopicassisted appendectomy and single-port laparoscopic appendectomy ${ }^{6}$ There are different trocar positioning in the three-port access. The surgeon usually operates on the left side of the patient, and the optic system is almost always introduced through an umbilical 5 or $10 \mathrm{~mm}$ port. The position of the two operating trocars may vary: triangulation with a right flank and mid-pelvic trocars, or both trocars positioned on the left flan. In both cases the scars remain visible, with a less aesthetic appeal. ${ }^{7}$

Several causes determine the high rate of laparotomic procedures for appendectomies, and among these are the following:

i. Cost of equipment and inputs used.

ii. Absence of systematization that does not require disposable and high-cost instruments, such as disposable trocars, staplers, bipolar or ultrasonic energy clamps, and disposable bags for extractors. We describe a technique in performing video laparoscopic appendectomy with three portals of low cost in inputs used. The incisions are located in positions that allow minimizing exposure of the scars, as long as they are located in the navel and iliac fossa. The materials are of permanent use, dispensing with any high-cost disposable input. We demonstrate the viability of this technique through its use in 1552 patients in a 20-year period. 


\section{Materials and methods} $1)$ :

Three punctures were performed to introduce the trocars (Figure

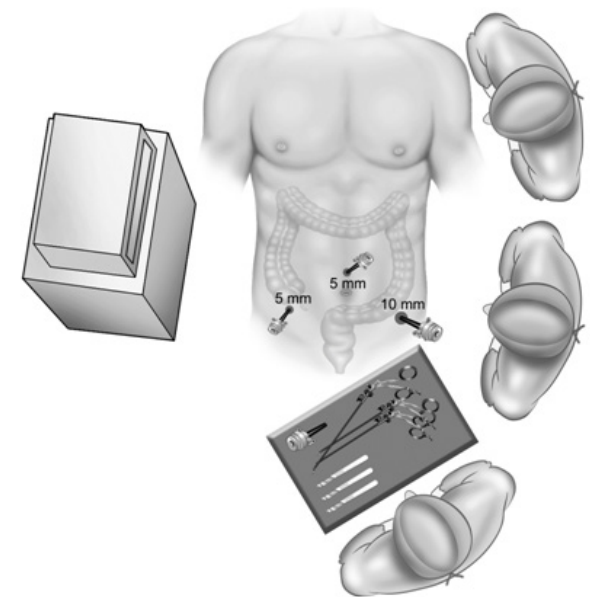

Figure I Positioning of the surgical team, punctures, and monitor.

a. The first puncture in the navel, 5 or $10 \mathrm{~mm}$ in diameter, for the placement of the telescope (depending on the available diameter) was performed with a permanent metal trocar. In the postoperative period, this incision is often imperceptible because it is located inside the navel.

b. Two punctures in the iliac fossae were located medial to the epigastric vessels. A 5-m-diameter permanent metal trocar on the right side and 10-mm-diameter permanent metal trocar with 5 -mm reducer on the left side were introduced. These punctures present quite satisfactory aesthetic results, as they are located in a position where they can be hidden by both male and female underwear. The surgeon positions on the left of the patient, with the assistant on his right and the instrumentation table on his left. The monitor was located to the right of the patient (Figure $1)$.

c. Surgery was performed with four permanent instruments: grasping forceps, hook, scissors, and needle holders. All these materials were resterilizable and of permanent use. A single 2-0 needle thread of cotton, silk, or Mersilene was used.

The operative technique consisted of the following steps:

i. An incision in the navel, grip of aponeurosis with forceps, and realization of the pneumoperitoneum with Veress needle. We use an intraabdominal pressure of $12 \mathrm{mmHg}$.

ii. 2. Introduction of a 5- or 10-mm permanent trocar, according to the availability of a 5- or 10-mm camera.

iii. Review of the abdominal cavity with the camera to identify other possible alterations and presence of liquid collections.

iv. Introduction of the 5-mm trocar in the right iliac fossa under direct vision, with the trocar being introduced medial to the epigastric vessels, which are visualized by transparency on the surface of the abdominal wall or by direct vision through the peritoneum inside the abdominal cavity.

v. Introduction of the $10-\mathrm{mm}$ trocar in the left iliac fossa, using the same method as that of the previous trocar. vi. Suction of liquid or purulent collections eventually present in the abdominal cavity.

vii. Patient positioning in Trendelenburg position and left lateral decubitus position for better exposure of the cecum region.

viii. Gripping the cecal appendix with the grasping forceps introduced by the right iliac fossa.

ix. With the hook inserted in the left iliac fossa trocar through a 5-mm metallic reducer, the cecal appendix is progressively separated from its meso, from its end to its base, with monopolar energy at $30 \%$ of its maximum limit. The cecum near the appendix is also released from epiploic appendages located near the appendicular base, mainly near the ileocecal valve, or even from the peritoneum, allowing the surface of the cecum peritoneum sufficient for a purse-string suture (Figure 2).

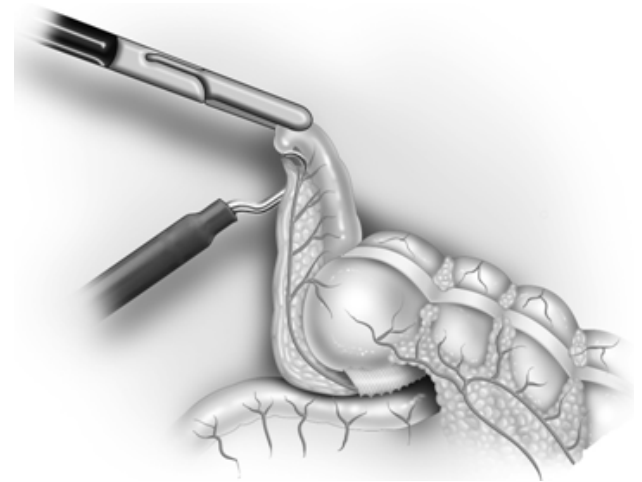

Figure 2 Release of the appendix of its meso with monopolar hook.

x. Suture of the base of the appendix with a 20-cm-long nonabsorbable 2-0 needle thread, transfixing the serosa in two points for better ligature fixation. Another more distal suture for the section of the appendix between the sutures is optional, decreasing the risk of an overflow of its contents. In the section of the suture thread, the rest of the needle thread remains in the abdominal cavity for future realization of the invagination bag of the appendicular stump.

xi. Grip (by the grasping forceps inserted, with reducer, in the 10$\mathrm{mm}$ trocar of the left iliac fossa) of the appendix (Figure 3).

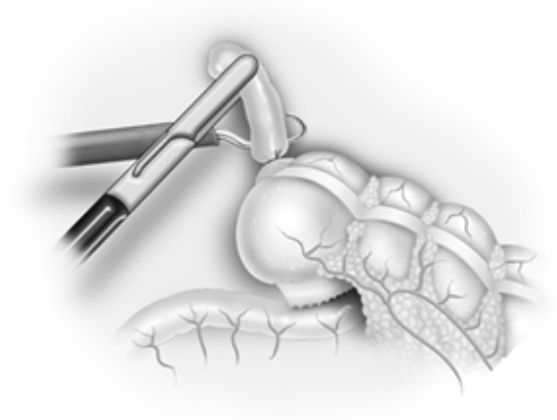

Figure 3 Appendix section next to the ligature with monopolar hook.

xii. Section of the appendix next to the suture performed at the base of the appendix (or between the two sutures, when the most distal suture is performed) using the hook introduced by the right iliac fossa (Figure 3). 
xiii. Removal of the seized appendix by pulling the grasping forceps immediately after the section into the $10-\mathrm{mm}$ trocar reducer (Figure 4).

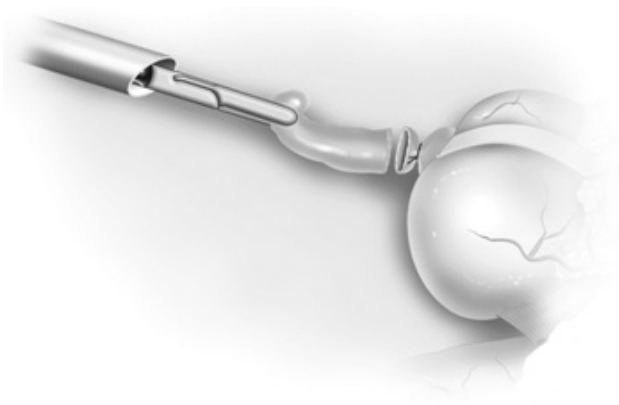

Figure 4 Pull and removal of the appendix through the left iliac fossa trocar immediately after its section, avoiding the use of an extraction bag.

xiv. In most cases, the diameter of the appendix (without its meso) allows its removal without the use of collector bags because, by pulling the grasping forceps into the reducer (Figure 5), the appendix remains inside the reducer, which is then pulled and removed without skin contact, avoiding contamination of the incision in the left iliac fossa. Almost all appendages, even quite inflamed, fit inside the $10-\mathrm{mm}$ trocar.

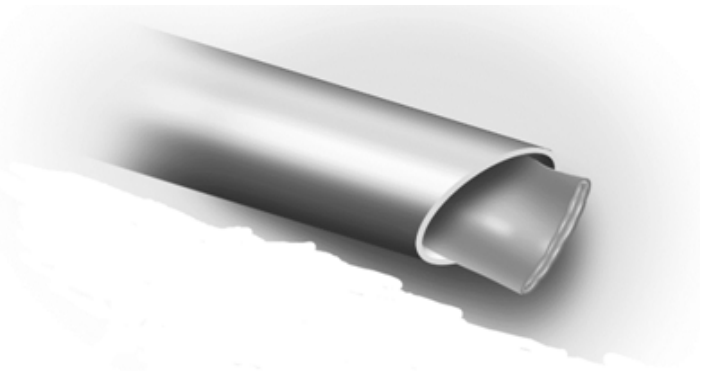

Figure 5 A 5-mm reducer of the left iliac fossa trocar with the cecal appendix inside.

$\mathrm{xv}$. The $10-\mathrm{mm}$ trocar is removed from the abdominal wall with the appendix inside. After the appendix is out, the trocar is inserted back into the abdominal wall.

xvi. This maneuver avoids the use of extraction bags that, besides increasing the cost of the procedure, require maneuvers to introduce the appendix inside, which requires time and increases the risk of contamination of the abdominal cavity.

xvii. Purse-string suture in the cecum around the appendicular stump for its occlusion using the rest of the 2-0 thread already present in the abdominal cavity.

\section{Results}

Surgery was performed in 1552 patients between 2000 and 2019, $56.25 \%$ of whom were women. The mean age of the patients was 32.66years (9-93years). The patients presented with appendicitis in all stages of evolution (Table 1) from edematous, purulent, or necrotic appendicitis. $\mathrm{n}$ four patients, necrosis of the appendix extended to the cecum, with inflammation and perforation in two patients, requiring right colectomy, which was also completed by a totally laparoscopic route. There were no conversions to open surgery or the need for insertion of additional trocars to perform appendectomy, except in cases of conversion to right colectomy. In all cases, inspection of the entire abdominal cavity and aspiration and washing, when there were purulent collections, were possible in all quadrants through the access used. Only 84 patients underwent drainage where there was an abscess in the right iliac fossa, using Silicone Penrose drain. Antibiotic prophylaxis was used in cases of edematous appendicitis with second-generation cephalosporin. In other patients, antibiotic therapy with third-generation cephalosporin and nitroimidazole was performed. The mean length of stay was 1.74 days (1-12days), with a median of 1.2 days. There were 6 rehospitalizations in the first 30days with significant abdominal pain or fever, and in 2 of these, there was a need for relaparoscopy for aspiration of purulent pelvic collection. Hyperemia and inflammation of the right iliac fossa incision developed in 74 patients $(4.7 \%)$, but no case of abscess that required drainage in the incisions was noted. There was no mortality.

Table I Staging of appendicitis

\begin{tabular}{lll}
\hline Appendicitis stages & Number of patients & Percentage \\
\hline Edematous & 821 & $52,9 \%$ \\
Purulent & 488 & $31,4 \%$ \\
Necrotic & 242 & $15,6 \%$ \\
\hline
\end{tabular}

\section{Discussion}

As described by Fitz and first performed in 1886 for the treatment of acute appendicitis, appendectomy is the safest treatment for this condition at any stage of its development. ${ }^{8}$ The incisions used for laparotomic access vary widely, but the most common is the access proposed by McBurney (oblique incision in the right iliac fossa). ${ }^{9}$ The aesthetic result is quite precarious when using laparotomic incisions, whether oblique, horizontal, or vertical. Most appendectomies are performed in children and adolescents, and aesthetics is an extremely important factor in the evaluation of the appendix extraction method. ${ }^{10}$ These scars will remain for life and can change as the patient grows, often becoming extremely unsatisfactory in appearance.

The introduction of the laparoscopic route for appendectomy, described by Kurt Semm in 1982, ${ }^{1}$ brought significant aesthetic benefits since it is almost always performed with three punctures, two of which are located in different positions in the abdominal wall but may be visible when the abdomen is exposed, depending on their location. This is of particular importance when the operation is performed on female adolescents.

The access through natural holes (excluding the navel from this classification) has not had a significant evolution, and even if it can be used in the future, it will require instruments and specialized equipment, increasing the cost of the procedure, in addition to requiring highly trained staff to perform it. ${ }^{11}$ The single umbilical access technique is feasible for appendectomy and has better aesthetic appeal in relation to multiple visible incisions in the anterior abdominal wall. ${ }^{8,12-18}$

For single access, the incision needs to be larger and may become visible or may deform the patient's navel. The incision must be at least $2.5 \mathrm{~cm}$ long for placement of a special trocar or three conventional trocars. ${ }^{11,19,20}$ Most appendectomy indications are present in children, adolescents, and young adults. An incision of this size can determine extremely precarious aesthetic results in these cases. ${ }^{21,22}$ Descriptions of single-access case series show that an additional trocar may be 
required in up to $10 \%$ of cases $^{23}$ or even be converted to a three-trocar technique, compromising the aesthetic aspect. ${ }^{15}$ In comparative series, it caused greater pain compared to conventional laparoscopy. ${ }^{24,25}$ The cosmetic result was considered better or comparable to the conventional method. ${ }^{8,12}$

Even if it has better aesthetic outcomes than conventional laparoscopy, the cost may be significantly higher because of the need to use special devices to introduce the instruments by single access. ${ }^{9,24,25}$ To reduce its cost, three trocars, 10 and $5 \mathrm{~mm}$ in diameter, can be placed together through a single enlarged umbilical incision. ${ }^{20,26}$ The descriptions of this strategy led authors, in comparative series, to consider the difficult execution and aesthetic final aspect similar to laparoscopy with three portals. ${ }^{16,17,23}$ In the single-portal technique, the technical difficulty of performing the dissection and section of the appendix with conventional instruments without triangulation and in a bad position to visualize the operative field cannot be disregarded, making the procedure more risky and more difficult to apply in the appendixes that are more difficult to resect. ${ }^{27,28}$

Safety is an important aspect to consider in surgery as common as appendectomy, where even the number of procedures performed by each surgeon has implications in increasing complications, length of hospital stay, and cost of the procedure..$^{27,29}$ In contrast, if performed safely and using a noninvasive method in a specialized environment, discharge rates can be achieved in less than a day in up to $90 \%$ of cases. ${ }^{6,30}$ The technique described in this study, with three portals - one umbilical and two supra-pubic - has an extremely satisfactory aesthetic result, because the scars remain hidden behind the underwear when the abdominal wall is exposed. The scar of the umbilical incision is small and may even be $5 \mathrm{~mm}$, not determining the deformation of the umbilical scar in any patient. The other two incisions remain hidden behind the patient's underwear.

This technique also allows appendectomy with a privileged view of the appendix and operative instruments. It allows adequate triangulation of the instruments, resulting in safety and shorter operative time of the procedure. The use of extremely close trocars can make triangulation difficult. ${ }^{31}$ In the described technique, the trocars are located in the iliac fossa, sufficiently apart to allow adequate triangulation.

The treatment of the mesoappendix, appendix, and stump is the subject of many studies. ${ }^{15,32}$ The use of endoclips, bipolar forceps, harmonic forceps, or staplers with vascular load is described in mesoappendix release. In all or almost all these treatment methods of the mesoappendix, it remains close to the appendix, greatly increasing the volume of the operative piece and forcing its extraction in special bags, which increases the cost and time of surgery. ${ }^{32,33}$ It should be noted that the removal of the mesoappendix is merely an artifice of surgical methods, as it can remain close to the cecum without any additional risk.

Moreover, by dissecting the meso next to the appendix, the technique described here makes ligation of the appendicular artery unnecessary, avoiding the use of devices for its occlusion and reducing the risk of bleeding. We use monopolar energy at $30 \%$ of the maximum level, dissecting the mesoappendix next to the cecal appendix, where the vessels are of smaller caliber. Performed in this way, the release of the appendix does not determine the risk of significant intraoperative bleeding. There was no need for reintervention for bleeding in any of our patients. The operative specimen, being only the inflamed appendix, can be removed in almost all cases through the $10-\mathrm{mm}$ trocar in the left iliac fossa, decreasing the risk of contamination of the abdominal wall during specimen removal (Figure 4).

If it is impossible for the trocar to remove the appendix, a small sterile plastic bag or an extremely low-cost piece of surgical glove is inserted through the incision of the left iliac fossa. The appendicular stump may only be sutured with 2-0 cotton thread and left exposed. Comparative studies between simple ligation and occlusion show no differences between the two methods of treatment of the appendicular stump. ${ }^{7}$ In the described technique, we performed a purse-string suture in the cecum around the appendicular stump and its invagination.

This procedure, being standardized and simplified, can be performed in any hospital environment that has a conventional laparoscopy system with the basic permanent forceps and shortens the operative time by quickly removing the piece with only one or two sutures. Because it does not use any instrument or disposable device, it has extremely low cost. ${ }^{34-37}$ It can be safely used at any age group, at any stage of appendicitis evolution, especially in morbidly obese individuals. We have systematically used this technique over the last 20years in 1552 patients who underwent surgery with low morbidity and absence of mortality. The access through three ports in all cases led to complete surgical resolution of the case, without the need for an additional trocar, attesting to its safety, efficiency, and replication. We hope with this contribution that video laparoscopic appendectomy will be more widely applied in the surgical treatment of acute appendicitis, since there is already concrete evidence about its superiority over laparotomy appendectomy, especially in more complicated cases or obese patients.

This study has several limitations. This is a retrospective study, and its results do not analyze all aspects of the patient's evolution. It aims to demonstrate that laparoscopic appendectomy can be performed at low cost without the use of disposable material and demonstrates the safety of the application of this systemization.

\section{Conclusion}

The technique we have described uses three metal trocars and four permanent instruments, with a single cotton thread. The use of operating pieces, extraction bags, clips, handles, staplers or special energy, and bipolar or harmonic instruments is discontinued. Therefore, it is an extremely low-cost laparoscopic procedure. Because it allows triangulation and instrumentation in the conventional way, it is a highly safe and reproducible surgery that can be easily taught and widely used in hospitals that have conventional laparoscopic equipment. The results obtained in 1552 patients, with low morbidity and absence of mortality, attest to the safety and reproducibility of the described access.

\section{Acknowledgments}

None.

\section{Conflicts of interest}

Author declares that there are no conflicts of interest.

\section{Funding}

None. 


\section{References}

1. Oliveira ALG, Oti AT, Yasojuma EY, et al. Laparoscopic appendectomy: prospective study of 300 cases. Arq Bras Cir Dig. 2008;21(2):48-52.

2. Slotboom T, Hamminga JT, Hofker HS, et al. Intraoperative motive for performing a laparoscopic appendectomy on a postoperative histological proven normal apêndix. Scand J Surg. 2014;103(4):245-248.

3. Borges PSGN, Lima MC, Falbo Neto GH. The Alvarado score validation in diagnosing acute appendicitis in children and teenagers at the Instituto Materno Infantil de Pernambuco, IMIP. Rev Bras Saude Mater Infant. 2003;3(4):439-445.

4. Gomes CA, Sartelli M, Podda M, et al. Laparoscopic versus open approach for diffuse peritonitis from appendicitis ethiology: a subgroup analysis from the Physiological parameters for Prognosis in Abdominal Sepsis (PIPAS) study. Updates Surg. 2020;72(1):185-191.

5. Brenner AS. Appendectomy in patients older than 40 years - analysis of the results of 217 cases. Rev Bras Colo-proctol. 2006;26(2):128-132.

6. Golebiewski A, Losin M, Murawski M, et al. One, two or three port appendectomy - a rational approach. Wideochir Inne Tech Malo Inwazijne. 2013;8(3):226-231.

7. Nicola Z, Gabriella S, Alberto M, et al. Transumbilical laparoscopicassisted appendectomy in children: clinical and surgical outcomes. World $J$ Gastrointest Endosc. 2014;6(4):101-104.

8. Villalobos-Mori R, Escoll-Rufino J, Herrerias-Gonzales F, et al. Prospective, randomized comparative study between singleport laparoscopic appendectomy and conventional laparoscopic appendectomy. Cir Esp. 2014;92(7):472-427.

9. Lasses-Martinez B, Ortiz-Oshiro E, Cabanas-Ojeda JL, et al. Cost is not a drawback to perform laparoscopic appendectomy in an academic hospital. Surg Laparosc Endosc Percutan Tech. 2014;24(2):24-29.

10. Cawich SO, Mohanty SK, Simpson LK, et al. Is laparoscopic appendectomy safe when performed in a low volumen setting? Int $J$ Biomed Sci. 2014;10(1):31-35.

11. Knuth J, Heiss MM, Bulian DR. Transvaginal hybrid-NOTES appendectomy in routine clinical use: prospective analysis of 13 cases and description of the procedure. Surg Endosc. 2014;28(3):731-739.

12. Clerveus M, Morandeira-Rivas A, Moreno-Sanz C, et al. Systematic review and meta-analysis of randomized controlled trials comparing single incision versus conventional laparoscopic appendectomy. World $J$ Surg. 2014;28:31-39.

13. Frazee RC, Abernathy SW, Davis M, et al. Outpatient laparoscopic appendectomy should be the standard of care for uncomplicated appendicitis. J Trauma Acute Care Surg. 2014;76(1):79-82.

14. Langer M, Safavi A, Skarsgard ED. Management of the base of the appendix in pediatric laparoscopic appendectomy: clip, lígate or staple? Surg Technol Int. 2013;23:81-83.

15. Lee JS, Hong TH. Comparison of various methods of mesoappendic dissection in laparoscopic appendectomy. J Laparoendosc Adv Surg Tech A. 2014;24(1):28-31.

16. Pinheiro RN, Sousa RC, Castro FM, et al. Single-incision videolaparoscopic appendectomy with conventional videolaparoscopic equipment. Arq Bras Cir Dig. 2014;27(1):34-37.

17. Qiu J, Yuan H, Chen S, et al. Single-port laparoscopic appendectomy versus conventional laparoscopic appendectomy: evidence from randomized controlled trials and nonrandomized comparative studies. Surg Laparosc Endosc Percutan Tech. 2014;24(1):12-21.

18. Sesia SB, Haecker FM. Laparoscopic-assisted single-port appendectomy in children: it is a safe and cost-effective alternative to conventional laparoscopic techniques? Minim Invasive Surg. 2013;8:24-29.
19. Bergholz R, Klein I, Wenke K, et al. Midterm outcome of transumbilically laparoscopic-assisted versus laparoscopic and open appendectomy. Europ J Pediatric Surg. 2014;28(3):35-39.

20. Raakow J, Klein D, Barutcu AG, et al. Single-port versus multiport laparoscopic surgery comparing long-term patient satisfaction and cosmetic outcome. Surg Endos. 2020.10.1007/s00464-019-07351-3.

21. Lasheen AE, Ezz R, Safwat K, et al. Minilaparoscopic appendectomy using a new spiral needle. Surg Endosc. 2014;28(3):645-649.

22. Minutolo V, Licciardello A, Di Stefano B, et al. Outcomes and cost analysis of laparoscopic versus open appendectomy for treatment of acute appendicitis. BMC Surg. 2014;14:14-20.

23. Neves LJVA. Simple ligation or ligation with bag making and burial for treatment of appendicular stump: a prospective randomized comparative study. Arq Bras Cir Dig. 2011;24(1):15-59.

24. Bergholz R, Krebs T, Klein I, et al. Transumbilical laparoscopic-assisted versus three-port laparoscopic and open appendectomy: a case-control study in children. Surg Laparosc Endosc Percutan Tech. 2014;24(3):244 247.

25. Carter JT, Kaplan JA, Nguyen JN, et al. A prospective, randomized controlled trial of single-incision laparoscopic vs conventional 3-port laparoscopic appendectomy for treatment of acute appendicitis. J Am Coll Surg. 2014;218(5):950-959.

26. Swank HA, van Rossem CC, van Geloven AA, et al. Endostapler or endoloops for securing the appendiceal stump in laparoscopic appendectomy: a retrospective cohort study. Surg Endosc. 2014;28(2):576-583.

27. Lee SE, Choi YS, Kim BG, et al. Single port laparoscopic appendectomy in children using glove port and conventional rigid instruments. Ann Surg Treat Res. 2014;86(1):35-38.

28. Gupta V, Singh SP, Bansal M, et al. Sutureless appendectomy by using harmonic scalpel: is it possible? J Laparoendosc Adv Surg Tech A. 2020. 10.1089/lap.2019.0782.

29. Kim CW, Jeon SY, Paik B, et al. Resident learning curve for laparoscopic appendectomy according to seniority. Ann Coloproctol. 2020.10.3393/ ac.2019.07.20

30. Cross W, Chadru Kowdley G. Same-day surgery for laparoscopic appendectomy in appendicitis: is this safe? Am Surg. 2014;80(1):25-26.

31. Gorter RR, Heij HA, Eker HH, et al. Laparoscopic appendectomy: State of the art. Tailored approach to the application of laparoscopic appendectomy? Best Pract Res Clin Gastroenterol. 2014;28(1):211-224.

32. Wright GP, Mitchell EJ, McClure AM, et al. Comparison of stapling techniques and management fo the mesoappendix in laparoscopic appendectomy. Surg Laparos Endosc Percutan Tech. 2014;24(5):315319.

33. Olijnyk JG, Pretto GG, da Costa Filho OP, et al. Two-port laparoscopic appendectomy as transition to laparoendoscopic single site surgery. $J$ Minim Access Surg. 2014;10(1):23-26.

34. Bessoff KE, Forrester JD. Appendicitis in low-resource settings. Surg Infec (Larchmt). 2020. 10.1089/sur.2019.265.

35. Shafy SZ, Miller R, Uffman JC, et al. An enhanced recovery protocol that facilitates same-day discharge for simple laparoscopic appendectomies. Pediatr Qual Saf. 2019;4(6):e243.

36. Birindelli A, Dester SE, Compagnoni B, et al. Retrograde technique with intra-corporeal tie in difficult laparoscopic appendectomy. Colorectal Dis. 2020. 10.1111/codi.14990.

37. Aguayo P, Alemayehu H, Desai AA, et al. Initial experience with same day discharge after laparoscopic appendectomy for nonperforated appendicitis. J Surg Res. 2014;4804(14):245-250. 